Confidential

\title{
Coatings superrepellent to ultralow surface tension liquids
}

Shuaijun Pan ${ }^{1,2^{*} \ddagger}$, Rui Guo ${ }^{1,2 \ddagger}$, Mattias Björnmalm ${ }^{2,4}$, Joseph J. Richardson ${ }^{2}$, Ling Li $^{1}$, Chang Peng $^{1,3}$, Nadja Bertleff-Zieschang ${ }^{2}$, Weijian $\mathrm{Xu}^{1 *}{ }^{*}$ Jianhui Jiang $^{1 *}$, Frank Caruso $^{2 *}$

${ }^{1}$ State Key Laboratory of Chemo/Biosensing and Chemometrics, College of Chemistry and Chemical Engineering, Hunan University, Changsha 410082, China.

${ }^{2} \mathrm{ARC}$ Centre of Excellence in Convergent Bio-Nano Science and Technology, and the Department of Chemical Engineering, The University of Melbourne, Parkville, Victoria 3010, Australia.

${ }^{3}$ College of Science, Hunan Agricultural University, Changsha 410128, China.

${ }^{4}$ Department of Materials, Department of Bioengineering, and the Institute of Biomedical Engineering, Imperial College London, London SW7 2AZ, UK.

*Correspondence to: shuaijun.pan@unimelb.edu.au (S.P.), weijxu@hnu.edu.cn (W.X.), jianhuijiang@hnu.edu.cn (J.J.), fcaruso@unimelb.edu.au (F.C.)

These authors contributed equally to this work. 


\begin{abstract}
High-performing coatings that durably and fully repel liquids are of interest for fundamental research and practical applications. Such coatings should allow for droplet beading, roll off and bouncing, which is difficult to achieve for ultralow surface tension liquids. Here, we report a bottom-up approach for preparing superrepellent coatings using a mixture of fluoro-silanes and cyanoacrylate. Upon application onto surfaces, the coatings assemble into thin films of locally multi-reentrant hierarchical structures with very low surface energy. The resulting material is superrepellent to solvents, acids and bases, polymer solutions and ultralow surface tension liquids, characterized by ultrahigh surface contact angles $\left(>150^{\circ}\right)$ and negligible roll-off angles $\left(\sim 0^{\circ}\right)$. Furthermore, the coatings are transparent, durable and demonstrate universal liquid bouncing, tailored responsiveness and anti-freezing properties, being thus a promising alternative to existing artificial superrepellent coatings.
\end{abstract}


Repellent coatings are of broad interest for investigating fundamental interfacial phenomena ${ }^{1-4}$, as well as for practical applications in areas such as self-cleaning ${ }^{5-7}$, chemical shielding ${ }^{8}$, heat transfer $^{3}$, wet adhesives ${ }^{9}$, drag-reduction $^{10}$, anti-fouling ${ }^{11}$, separations and membranes ${ }^{12,13}$, fogharvesting $^{14}$, self-assembly ${ }^{15}$, and icephobicity and anti-freezing ${ }^{2,16,17}$. Combining sophisticated microstructures possessing reentrant ${ }^{18}$ or double-reentrant textures ${ }^{19}$ with low surface energy chemical modifications ${ }^{20-22}$ result in state-of-the-art techniques for the preparation of repellent surfaces (i.e. surfaces with apparent contact angles $\theta^{*}>150^{\circ}$, which are considered superhydrophobic or superoleophobic for water and oil, respectively). However, engineering high-performing surfaces that are superrepellent (i.e. droplet roll-off angles $\sim 0^{\circ}$ ) even to liquids with ultralow surface tensions (i.e. $<20 \mathrm{mN} \mathrm{m}^{-1}$; e.g., $n$-hexane and $n$-pentane) remains challenging because of their low solid-liquid interfacial energy (see Supplementary Figs. 1 and 2 for detailed discussion). To date, this has only been possible using a "top-down", multi-step etching-based approach ${ }^{19}$, which can have limited applicability and versatility due to a lack of robustness, e.g., break-in of liquid.

\section{Strategy}

The overall performance of a coating is governed by a range of properties ${ }^{23}$-including surface morphology, binding forces, surface chemistry, and other physical and mechanical characteristics — whereas surface repellence to liquids is primarily dependent on the surface texture and chemistry ${ }^{20,24}$. As these factors are interrelated, the design of simple and versatile superrepellent coatings is difficult. For example, increasing surface roughness may be used to minimize the liquid-solid contact area, which favours ultrahigh contact angles and ultralow rolloff angles. However, increased surface roughness can also compromise the transparency and durability of a coating ${ }^{20,25}$. Additionally, the responsiveness of a coating may enable surfaces 
with switchable and self-healing capabilities, but this "active" nature can reduce durability and performance of the coating over repeated $\operatorname{cycles}^{26-28}$. Diverse strategies have been reported for preparing films with properties such as transparency ${ }^{25,29,30}$, mechanical durability ${ }^{6,27}$, selfhealing ${ }^{31}$, or switchability of wetting states (i.e. between wetted Wenzel ${ }^{32}$ and non-wetted Cassie-Baxter ${ }^{33}$ states $^{34}$ that can be sustained even when exposed to dynamic contact (e.g., strains or impinging drops) ${ }^{29,35}$ or harsh temperatures (e.g., during freezing) $)^{19,22,36}$. Although these studies demonstrate that repellent materials with different properties can be made, a universal coating technique combining all of these capabilities (i.e. repelling targeted contacting liquids — superomniphobicity—without compromising other functionalities) is yet to be reported.

Herein, we report a "bottom-up" approach ${ }^{37-40}$ to prepare coatings that are superrepellent to a range of liquids $(>100)$, including liquids with surface tensions of less than $20 \mathrm{mN} \mathrm{m}^{-1}$ and even liquefied gases (e.g., liquid nitrogen, $8.8 \mathrm{mN} \mathrm{m}^{-1}$ ). The coatings can be assembled using immersion assembly and spray assembly, two well-established technologies used for preparing nanofilms ${ }^{23}$. The superrepellent coatings are assembled through the formation of multi-reentrant nano- and microstructures displaying ultralow surface energy through vapour-induced hydration and condensation of fluoro-silanes and cross-linking of medical-grade cyanoacrylates. These nanocoatings are locally hierarchically structured, chemically bonded, and can be applied to a wide range of substrates (e.g., flexible or rigid, flat or textured, transparent or opaque). The coatings are largely transparent, robust, mechanically durable, self-healing, and demonstrate substrate-mediated versatility through wettability, responsiveness, reduced contact time, remarkable impact robustness for impinging droplets and liquid jets, and enhanced anti-freezing and ice-removal performance, thus extending the capabilities — and our understanding — of artificial superrepellent coatings. 


\section{Repellent coatings}

First, we investigated the assembly and performance of the studied films by spray-coating plastic surfaces. The spray-coating solution was created by simply mixing $1 \mathrm{H}, 1 \mathrm{H}, 2 \mathrm{H}, 2 \mathrm{H}-$ perfluorohexyltrichlorosilane (PFTS) and $n$-butyl cyanoacrylate ( $n$-BCA) ( $n$-BCA is commonly used for medical adhesives) in dichloropentafluoropropane solvent (Asahiklin 225). We note that, though rare, dichloropentafluoropropane could etch or dissolve some weak plastics such as poly(methyl methacrylate). This may be beneficial (e.g., for anchoring the coating to the substrate) or detrimental, depending on the desired application. Polydimethylsiloxane (PDMS) discs, a flexible and transparent material, were chosen as substrates to demonstrate the transparency of the superomniphobic (SOP) coatings. After spray-coating, the PDMS surfaces displayed hierarchical textures composed of trapezoidal structures with multi-reentrant curvatures (Fig. 1a,b). As shown in Fig. 1c, the high fluorination degree of cross-linked PFTS makes the coating one of the lowest surface energy materials known to date $\left(10 \mathrm{mN} \mathrm{m}^{-1}\right)^{18}$ (see Supplementary Figs. 1 and 2 for detailed discussion). Owing to the local multi-reentrant structures and ultralow surface energy of the thinly spray-coated layer, the PDMS surfaces were both transparent and superrepellent to a broad range of liquids of different polarities, surface tensions, and corrosiveness (Fig. 1d and Supplementary Table 1), as exemplified by the ultrahigh contact angle of an $n$-hexane droplet (inset in Fig. 1a).

The influence of coating conditions on the resulting properties (e.g., optical transmittance, surface morphology, phase separation and wetting behaviour) was investigated (Fig. 1e; Supplementary Figs. 3-8). Using 20\% PFTS and a spray time of 4 min, a coating that could transmit $95 \%$ of visible light while remaining superrepellent, even to $n$-hexane, was prepared. $n$ Hexane has an extremely low surface tension $\left(17.9 \mathrm{mN} \mathrm{m}^{-1}\right.$, Supplementary Tables 1 and 2), and 
even for surfaces that demonstrate a high contact angle to $n$-hexane, it often remains adhered to the surface ${ }^{28,41}$. Therefore, highly specialized procedures have previously been required to produce surfaces that are superrepellent to $\mathrm{it}^{19}$. Using a high-speed camera, we showed that $n$ hexane exhibited high dynamic contact angles to the spray-coated material and that it bounced five times before residing on the coating (Fig. 1f; Supplementary Movie 1). Droplet bouncing using liquids with low surface tensions is a hallmark of high-performing superrepellent surfaces ${ }^{19,20}$.

We then investigated the versatility of spray coating using different substrates. The use of $n$ BCA as a binder not only trapped cross-linked PFTS within the coating, but also strongly anchored the composite to all substrates tested (Supplementary Figs. 9 and 10; 24 substrates were tested, including metals, polymers, glass, fabric, wood, and composites) regardless of their inherent hydrophobicity or hydrophilicity (see crosslinking details in Supplementary Figs. 9-12). This versatility is due to the excellent binding nature of the mixture once sprayed and exposed to moisture in the air (i.e. presence of water vapour, ammonia, acid, and heat can promote the crosslinking reactions). This result demonstrates the versatility and near universal and substrateindependent properties of this technique. However, the morphology of the substrate could affect the local area contact with a probing liquid — rougher substrates essentially increased the hierarchy of a coating, which minimized the local liquid contact area, resulting in more robust repellency.

\section{Hierarchical effect}

Next, we investigated the possibility of leveraging substrate hierarchy to further enhance the superrepellency of the coated material. By comparing dip- and spray-coating for commercially 
available high-quality meshes, polyester fabrics, and flat silicon wafers, more than five levels of surface features could be engineered and the resulting wettabilities were studied (see Supplementary Figs. 13 and 14 for detailed discussion). The best performing non-wetting capabilities were observed for the spray-coated fabric (the "highest-level" material), as expected (Fig. 2a-d). The most robust coatings were obtained after spray-coating onto a fully stretched fabric ( $\sim 100 \%$ increase in length from resting, non-stretched state), to facilitate access between fibres, which resulted in even denser coatings once released (Fig. 2a,c; textural and inter-fibre spacings are $\sim 100 \mu \mathrm{m}$ and $\sim 10 \mu \mathrm{m}$, respectively; diameters of the textural fibre bundle and individual fibres are $\sim 200 \mu \mathrm{m}$ and $\sim 20 \mu \mathrm{m}$, respectively). All liquids tested were observed to bead up on top of the fabric, even $n$-pentane, which has one of the lowest surface tensions known (15.5 $\mathrm{mN} \mathrm{m}^{-1}$, Supplementary Tables 1 and 2) (Fig. 2b,d). This behaviour is assumed to originate from the five levels of hierarchy of the surface (Fig. 2c) and the low-energy nature of the coating (Supplementary Figs. 1 and 2). Moreover, the combination of these properties provides ultra-small local contact areas with liquids. This is shown by the ultrahigh contact angles and ultralow roll-off angles obtained for the probe liquids (>100) tested (Fig. 2d; Supplementary Table 2), which covered a broad range of surface tensions $\left(14.0-98.0 \mathrm{mN} \mathrm{m}^{-1}\right)$ and included corrosive acids and bases (e.g., concentrated $\mathrm{HF}, \mathrm{HNO}_{3}, \mathrm{H}_{2} \mathrm{SO}_{4}$, and $\mathrm{KOH}$ ).

To explore the theory underlying the role of the hierarchical textures, a recursive form ${ }^{42}$ of the Cassie-Baxter relation was used to evaluate the ultrahigh contact angles (see Supplementary Fig. 14 for detailed discussion). The results suggest a key role of hierarchically reentrant textures in repelling ultralow surface tension liquids (i.e. significantly reduced liquid-solid contact area favours the beading up of the liquid, which further promotes droplet rolling and bouncing with ultra-low contact angle hysteresis $)^{42-47}$. Surface chemistry is also important. For instance, oxygen 
plasma treatments are commonly used to decompose various surface chemistries (e.g., to convert non-wetting surfaces into wetting surfaces) (Fig. 2e). Interestingly, the superrepellent properties of the coating introduced here could be simply restored by either heat treatment $\left(120^{\circ} \mathrm{C}, 10 \mathrm{~min}\right)$ or storage at ambient temperature $\left(20-23{ }^{\circ} \mathrm{C},>24\right.$ h) (Fig. 2e,f; Supplementary Figs. 15 and 16). This self-healing property is assumed to result from the preferential phase segregation (Supplementary Fig. 4) of cross-linked PFTS from the surface of the coating, which is thermodynamically favourable because it minimizes the overall free energy ${ }^{8}$. Furthermore, the self-restored superrepellency was evident from the hysteresis-free locomotion of a pentane droplet rolling off the coated Anticon fabric shown in Fig. 2a when given arbitrary slope angles of $0^{\circ}<\alpha<90^{\circ}$ (Fig. 2g; Supplementary Movies 2 and 3). By considering the forces acting on the droplet (gravity and hysteresis arising from advancing and receding local contact interfaces) ${ }^{28}$, theoretical rolling profiles can be compared with the results obtained, and the profiles were found to be largely in agreement with that of a friction-free model surface (i.e., the coating is virtually friction-free assuming negligible contact angle hysteresis) (Fig. 2h). Moreover, gently impinging pentane droplets (i.e. low Weber number, e.g., $W e=10 ; W e$ is defined as the ratio between kinetic energy and the surface energy of the droplet $W e=\rho R_{0} V^{2} / \gamma$, where $\rho, \gamma, R_{0}$ and $V$ are the density, surface tension, radius and velocity of the impinging droplet, respectively) bounced freely on the coated fabric, and more than six rebounds were observed (Fig. 2i; Supplementary Movie 4). These results demonstrate the occurrence of ultralow contact angle hysteresis and exemplify the most superrepellent coating reported to date.

\section{Leveraging robustness}


The robustness of the coating and possibility of creating switchable and responsive surfaces were investigated by using the stretchability of the fabric (Fig. 3 and see Supplementary Figs. 17 and 18 for detailed discussion). By spray-coating the fabric in the relaxed state and stretching subsequently, superomniphilic (SOL) and SOP regions could be prepared within the same fabric (Fig. 3d). The accessibility of local regions of superomniphilicity depends on both the scale of the area and how easily a droplet penetrates. To investigate this, a dimensionless robustness factor $\left(A^{*}\right)$ can be used ${ }^{42,48}$ :

$$
A^{*}=\frac{l_{\text {cap }}}{R(\zeta d+d-1)} \frac{1-\cos \theta}{(\zeta d+d-1+2 \sin \theta)}
$$

where $A^{*}$ represents the ratio between the wetting pressure and a droplet's minimum pressure across the composite interface and is a function of the capillary length $l_{\text {cap }}=\sqrt{\gamma / \rho g}(\rho$ is density, $\gamma$ is surface tension, and $g$ is acceleration of gravity). Higher $A^{*}$ values indicate that the coating is more robust (i.e. SOP), and the SOL state becomes more accessible as $A^{*}$ decreases. By defining the surface spacing ratio $d=1+D / R$ (where $R$ and $D$ represent the mean radius of the fabric bundles and interspacing, respectively), one can evaluate whether a coating is more likely or not to wet by a given liquid with Young's angle $\theta$ at given elongation $\zeta$. With this as a foundation, the wetting transitions of pentane and water for partially spray-coated fabrics were investigated (Fig. 3e). The SOP fabric was least accessible by water (i.e. wetting transition only occurs when $\zeta$ approaches 100\%). Considering its high surface tension and Young's angle, this observation highlights the difficulties associated with fabricating surfaces that completely repel low surface tension liquids such as alkanes. For further investigation, the robustness factors $A^{*}$ for seven $n$-alkanes $\left(\mathrm{C}_{5}, \mathrm{C}_{6}, \mathrm{C}_{7}, \mathrm{C}_{8}, \mathrm{C}_{10}, \mathrm{C}_{12}, \mathrm{C}_{16}\right)$ were evaluated and experimentally tested (Supplementary Fig. 18). These low surface tension liquids could penetrate the fabric readily 
even at small strains (e.g., $\zeta \approx 20 \%$ for pentane) (Fig. 3e and Supplementary Fig. 18).

Furthermore, the wetting transition of these partially spray-coated fabrics was reversible (Fig. 3f) owing to the stretchability of the material. Even after 1,000 cycles of stretch-release tests, the relaxed material remained superrepellent (Supplementary Fig. 19), thus demonstrating the robustness and mechanical durability of the coating. These features are important for developing responsive surfaces, for example, for the selective separation of oil/water mixtures ${ }^{28}$.

When comparing partially coated fabrics (spray-coated while relaxed, so that only surfaceexposed fibres in the fabric are coated) with fully coated fabrics (spray-coated while stretched so that coating can penetrate between fibres in the fabric), distinct liquid repelling performances were observed. Partially coated fabrics, though superrepellent for low surface tension static droplets, could be wetted by impacting oil droplets of medium $W e$ values $(W e=90)$ (Supplementary Fig. 20a and Supplementary Movie 5). Contrarily, the stretch-coated fabric displayed surprising repellence even to a pentane drop impacting at the same $W e$ value (Supplementary Fig. 20b and Supplementary Movie 6). This result can be attributed to the complete coating of the fibre bundles and areas between the fibres. At $W e$ values greater than 90 (e.g., $W e=160$ ), pentane droplets bounced off the substrate, breaking into smaller droplets (i.e. satellite droplets). This result can be attributed to the low surface tension and viscosity of the fluids tested (Fig. 3g; Supplementary Movie 7). Driven by the superrepellent nature of the fully coated fabric, the small droplets bounced several times before residing on the surface, implying that the area impacted by the original droplet remained intact (the temporal "break-in" of the droplet into the material does not actually break through the superrepellent coating, i.e. wet the material). For even stronger impacts (e.g., $W e=250$ ), several satellite droplets were observed to break off from the original droplet and bounced off the surface before coalescing 
(Supplementary Fig. 21 and Supplementary Movie 8), however, without wetting the material, thus demonstrating the high robustness of the coating. Further robustness studies revealed that the superrepellency of the coating survives $>100,000$ impacts of pentane droplets released at a height of $\sim 30 \mathrm{~cm}$ above the surface (i.e. the impact pressure was $\sim 0.3 \mathrm{MPa}$ ), and no liquid breakthrough was observed even when the fabric was positioned directly underneath a $\sim 100 \mathrm{~cm}$ liquid column of pentane (i.e. the hydrostatic pressure was $\sim 6,000 \mathrm{~Pa}$ ) (Supplementary Fig. 22). Also, no liquid breakthrough was observed when continuously impacting a coated substrate with a pentane jet (e.g., rate of flow $\sim 1 \mathrm{~mL} \mathrm{~s}^{-1}$, Supplementary Movie 9).

\section{Air cushioning}

Owing to the combined effect of the multi-reentrant texture and the low surface energy of the material, the sprayed coating can also repel fluorinated solutions (e.g., concentrated HF and polyvinylidene fluoride solutions, Supplementary Fig. 23, Supplementary Movies 10 and 11) as well as fluorine-based liquids ${ }^{19}$ (e.g., perfluorohexane known as FC-72, $10 \mathrm{mN} \mathrm{m}^{-1}$ ) at elevated temperatures (e.g., $>100^{\circ} \mathrm{C}$ ) (Supplementary Fig. 24; Supplementary Movie 12). The bouncing performance of these liquids might also be ascribed to the continuous presence of an air film ${ }^{49}$ between the impinging droplet and the coating surface, resulting from the Leidenfrost effect ${ }^{3}$ and/or from geometry effects of the multi-reentrant textures ${ }^{19}$. It is worth noting that the bouncing of FC-72 can be promoted at room temperature (e.g., $\left.20^{\circ} \mathrm{C}\right)$ given increased ambient pressures (e.g., bouncing in a chamber filled with pressured nitrogen, Supplementary Fig. 24 and Supplementary Movie 13). To maintain superrepellency to low surface tension liquids, a gas cushion must be present to slow down the incoming liquid droplets and reverse their momentum ${ }^{49}$. A higher density of the gas medium at the surface thus decreases the chance of gas drainage and subsequent local wetting ${ }^{50}$. By investigating the surface wettability of a wide 
spectrum of liquids ( $>100)$, we have found that most of the liquids tested roll off the coated substrates at small tilt angles (e.g., $<5^{\circ}$, Fig. 4 a and Supplementary Fig. 25) demonstrating ultralow adhesion with the surfaces. Furthermore, the sprayed coatings were even found to repel liquid nitrogen (Fig. 4b; Supplementary Movies 14-16). By comparison, the uncoated porous substrates displayed superwetting under these cryogenic conditions (Supplementary Figs. 26 and 27; Supplementary Movie 17). We hypothesize that the superrepellent coatings maintain a stable gas layer at the surface, which also helps to keep the Leidenfrost effect ${ }^{3}$ once the evaporation takes place at the liquid-solid interface.

Moreover, the superrepellency provided by the hierarchical structures can minimize the local liquid-solid contact, due to the presence of the air cushion, and thus reduces heat transfer at the liquid-solid interface. Building on this, we have further explored anti-freezing, low ice adhesion, and robust liquid bouncing (i.e. reduced contact time and universal bouncing of a broad spectrum of liquids) properties of the hierarchically multi-reentrant superrepellent surfaces (see Supplementary Figs. 28-35, Supplementary Movies 18-25, and Supplementary Table 3 for detailed discussions). The observed liquid bouncing dynamics suggests a new bouncing mechanism ("composite pancake bouncing"; Supplementary Figs. 33-35, and Supplementary Movies 24 and 25).

\section{Outlook}

In addition to the facile and versatile spray-coating application of the superrepellent film, an important factor for practical applications is durability ${ }^{27}$. Superrepellency becomes more challenging at elevated temperatures as the surface tension of the contacting liquid decreases under such conditions. However, the coated fabrics retained their non-wetting capability with no 
observed increase in roll-off angles at testing temperatures of $1-100{ }^{\circ} \mathrm{C}$ using water and $n$-octane (Supplementary . 36). In addition to the aforementioned durability tests, the mechanical durability of the coating was also tested — the coating could withstand scratch, shear, abrasion, peeling, sonication, and washing, while exhibiting negligible decreases in performance (Supplementary Fig. 36 and Supplementary Movie 26). It is worth noting that versatile coatings could be easily engineered by incorporating additional desired components into the system (e.g., introducing a third material into the coating, Supplementary Fig. 37).

\section{References}

1. Li, Y., Quere, D., Lv, C. \& Zheng, Q. Monostable superrepellent materials. Proc. Natl. Acad. Sci. U.S.A. 114, 3387-3392 (2017).

2. Schutzius, T. M. et al. Spontaneous droplet trampolining on rigid superhydrophobic surfaces. Nature 527, 82-85 (2015).

3. Vakarelski, I. U., Patankar, N. A., Marston, J. O., Chan, D. Y. \& Thoroddsen, S. T. Stabilization of Leidenfrost vapour layer by textured superhydrophobic surfaces. Nature 489, 274-277 (2012).

4. Yong, J., Chen, F., Yang, Q., Huo, J. \& Hou, X. Superoleophobic surfaces. Chem. Soc. Rev. 46, 4168-4217 (2017).

5. Blossey, R. Self-cleaning surfaces - virtual realities. Nat. Mater. 2, 301-306 (2003).

6. Lu, Y. et al. Robust self-cleaning surfaces that function when exposed to either air or oil. Science 347, 1132-1135 (2015).

7. Erbil, H. Y., Demirel, A. L., Avci, Y. \& Mert, O. Transformation of a simple plastic into a superhydrophobic surface. Science 299, 1377-1380 (2003).

8. Pan, S., Kota, A. K., Mabry, J. M. \& Tuteja, A. Superomniphobic surfaces for effective chemical shielding. J. Am. Chem. Soc. 135, 578-581 (2013).

9. Lee, H., Lee, B. P. \& Messersmith, P. B. A reversible wet/dry adhesive inspired by mussels and geckos. Nature 448, 338-341 (2007).

10. N'Guessan, H. E. et al. Water tribology on graphene. Nat. Commun. 3, 1242 (2012). 
11. Epstein, A. K., Wong, T.-S., Belisle, R. A., Boggs, E. M. \& Aizenberg, J. Liquid-infused structured surfaces with exceptional anti-biofouling performance. Proc. Natl. Acad. Sci. U. S. A. 109, 13182-13187 (2012).

12. Kota, A. K., Kwon, G., Choi, W., Mabry, J. M. \& Tuteja, A. Hygro-responsive membranes for effective oil-water separation. Nat. Commun. 3, 1025 (2012).

13. Han, Y., Xu, Z. \& Gao, C. Ultrathin graphene nanofiltration membrane for water purification. Adv. Funct. Mater. 23, 3693-3700 (2013).

14. Zheng, Y. et al. Directional water collection on wetted spider silk. Nature 463, 640-643 (2010).

15. Timonen, J. V. I., Latikka, M., Leibler, L., Ras, R. H. A. \& Ikkala, O. Switchable static and dynamic self-assembly of magnetic droplets on superhydrophobic surfaces. Science 341, 253-257 (2013).

16. Bird, J. C., Dhiman, R., Kwon, H.-M. \& Varanasi, K. K. Reducing the contact time of a bouncing drop. Nature 503, 385-388 (2013).

17. Kreder, M. J., Alvarenga, J., Kim, P. \& Aizenberg, J. Design of anti-icing surfaces: smooth, textured or slippery? Nat. Rev. Mater. 1, 15003 (2016).

18. Tuteja, A. et al. Designing superoleophobic surfaces. Science 318, 1618-1622 (2007).

19. Liu, T. \& Kim, C. J. Turning a surface superrepellent even to completely wetting liquids. Science 346, 1096-1100 (2014).

20. Deng, X., Mammen, L., Butt, H.-J. \& Vollmer, D. Candle soot as a template for a transparent robust superamphiphobic coating. Science 335, 67-70 (2011).

21. Wong, T.-S. et al. Bioinspired self-repairing slippery surfaces with pressure-stable omniphobicity. Nature 477, 443-447 (2011).

22. Azimi, G., Dhiman, R., Kwon, H.-M., Paxson, A. T. \& Varanasi, K. K. Hydrophobicity of rare-earth oxide ceramics. Nat. Mater. 12, 315-320 (2013).

23. Richardson, J. J., Björnmalm, M. \& Caruso, F. Technology-driven layer-by-layer assembly of nanofilms. Science 348, aaa2491 (2015).

24. Steele, A., Bayer, I. \& Loth, E. Inherently superoleophobic nanocomposite coatings by spray atomization. Nano Lett. 9, 501-505 (2009).

25. Zhang, J. \& Seeger, S. Superoleophobic coatings with ultralow sliding angles based on silicone nanofilaments. Angew. Chem. Int. Ed. 50, 6652-6656 (2011). 
26. Stuart, M. A. C. et al. Emerging applications of stimuli-responsive polymer materials. Nat. Mater. 9, 101-113 (2010).

27. Tian, X., Verho, T. \& Ras, R. H. A. Moving superhydrophobic surfaces toward realworld applications. Science 352, 142-143 (2016).

28. Pan, S., Guo, R. \& Xu, W. Durable superoleophobic fabric surfaces with counterintuitive superwettability for polar solvents. AIChE J. 60, 2752-2756 (2014).

29. Yao, X. et al. Adaptive fluid-infused porous films with tunable transparency and wettability. Nat. Mater. 12, 529-534 (2013).

30. Wong, W. S. Y. et al. Omnidirectional self-assembly of transparent superoleophobic nanotextures. ACS Nano 11, 587-596 (2017).

31. Xu, Z., Zhao, Y., Wang, H., Wang, X. \& Lin, T. A superamphiphobic coating with an ammonia-triggered transition to superhydrophilic and superoleophobic for oil-water separation. Angew. Chem. Int. Ed. 54, 4527-4530 (2015).

32. Wenzel, R. N. Resistance of solid surfaces to wetting by water. Ind. Eng. Chem. 28, 988994 (1936).

33. Cassie, A. B. D. \& Baxter, S. Wettability of porous surfaces. Trans. Faraday Soc. 40, 546-551 (1944).

34. Bormashenko, E., Pogreb, R., Balter, S. \& Aurbach, D. Electrically controlled membranes exploiting Cassie-Wenzel wetting transitions. Sci. Rep. 3, 3028 (2013).

35. Hao, C. et al. Superhydrophobic-like tunable droplet bouncing on slippery liquid interfaces. Nat. Commun. 6, 7986 (2015).

36. Jung, S., Tiwari, M. K., Doan, N. V. \& Poulikakos, D. Mechanism of supercooled droplet freezing on surfaces. Nat. Commun. 3, 615 (2012).

37. Golovin, K. et al. Designing durable icephobic surfaces. Sci. Adv. 2, e1501496 (2016).

38. Campos, R. et al. Superoleophobic surfaces through control of sprayed-on stochastic topography. Langmuir 28, 9834-9841 (2012).

39. Golovin, K., Lee, D. H., Mabry, J. M. \& Tuteja, A. Transparent, flexible, superomniphobic surfaces with ultra-low contact angle hysteresis. Angew. Chem. Int. Ed. 52, 13007-13011 (2013). 
40. Srinivasan, S., Chhatre, S. S., Mabry, J. M., Cohen, R. E. \& McKinley, G. H. Solution spraying of poly(methyl methacrylate) blends to fabricate microtextured, superoleophobic surfaces. Polymer 52, 3209-3218 (2011).

41. Grigoryev, A., Tokarev, I., Kornev, K. G., Luzinov, I. \& Minko, S. Superomniphobic magnetic microtextures with remote wetting control. J. Am. Chem. Soc. 134, 1291612919 (2012).

42. Kota, A. K., Li, Y., Mabry, J. M. \& Tuteja, A. Hierarchically structured superoleophobic surfaces with ultralow contact angle hysteresis. Adv. Mater. 24, 5838-5843 (2012).

43. Verho, T. et al. Reversible switching between superhydrophobic states on a hierarchically structured surface. Proc. Natl. Acad. Sci. U. S. A. 109, 10210-10213 (2012).

44. Herminghaus, S. Roughness-induced non-wetting. Europhys. Lett. 52, 165-170 (2000).

45. Kleingartner, J. A. et al. Designing robust hierarchically textured oleophobic fabrics. Langmuir 31, 13201-13213 (2015).

46. Mazumder, P. et al. Superomniphobic, transparent, and antireflection surfaces based on hierarchical nanostructures. Nano Lett. 14, 4677-4681 (2014).

47. Jung, Y. C. \& Bhushan, B. Mechanically durable carbon nanotube-composite hierarchical structures with superhydrophobicity, self-cleaning, and low-drag. ACS Nano 3, 4155-4163 (2009).

48. Tuteja, A., Choi, W., McKinley, G. H., Cohen, R. E. \& Rubner, M. F. Design parameters for superhydrophobicity and superoleophobicity. MRS Bull. 33, 752-758 (2008).

49. de Ruiter, J., Lagraauw, R., van den Ende, D. \& Mugele, F. Wettability-independent bouncing on flat surfaces mediated by thin air films. Nat. Phys. 11, 48-53 (2014).

50. Qian, J. \& Law, C. K. Regimes of coalescence and separation in droplet collision. J. Fluid Mech. 331, 59-80 (1997). 


\section{Methods}

Master coatings. Solutions $\left(10 \mathrm{mg} \mathrm{mL}^{-1}\right)$ of $n$-BCA (BOC Sciences) $+20 \mathrm{wt} \% \%$ PFTS (Gelest) were prepared in Asahiklin 225 (Asahi Glass Co.). Transparent coatings were prepared by spraying PDMS discs for 0-10 min using a Paasche airbrush at a distance of $\sim 20 \mathrm{~cm}$ with a $\mathrm{N}_{2}$ pressure of 58 psi. Hierarchically structured surfaces were obtained by spraying Anticon polyester fabric (Ultrapure Technology) (5-tier) and stainless steel (SS) meshes (McMaster-Carr) (4-tier) for $5 \mathrm{~min}$. To ensure complete penetration of the coatings into the fabric, Anticon was $100 \%$ biaxially elongated for spraying. (If used in a relaxed, non-stretched state, only a facial coating will form). Surfaces with a coarser length-scale texture were prepared by dip-coating the polyester fabric (4-tier) and SS mesh (3-tier micro) for 5 min. 3-Tier nanostructures could be prepared by spraying silicon wafers for 2 min (thus avoiding larger particle aggregates). Dipcoated silicon wafers were also used to estimate the solid surface energy of PFTS+BCA complexes (i.e. the ratio of PFTS varied from 0 to 100\%). Non-textured surfaces (2-tier) were prepared by dip-coating silicon wafers for $5 \mathrm{~min}$. All coated surfaces were subsequently crosslinked at $70{ }^{\circ} \mathrm{C}$ for $2 \mathrm{~h}$. PFTS-deposited silicon wafers were prepared as comparison (1-tier). Chemical vapor deposition was performed by placing wafers together with $10 \mu \mathrm{L}$ PFTS on a 70 ${ }^{\circ} \mathrm{C}$ hot plate for $2 \mathrm{~h}$. The silanized wafers were subsequently washed with ethanol and dried under $\mathrm{N}_{2}$ stream. Plain silicon wafers (hydrophilic) were also used as controls (e.g., in freezing and ice adhesion tests). All the above surfaces were stored in a vacuum desiccator before usage.

Goniometer. The contact angle, roll-off angle, and surface tension measurements were conducted using a Ramé-Hart 200-F1 goniometer. Contact angles measurements were implemented by advancing and receding a small droplet of liquid $(\sim 5 \mu \mathrm{L})$ onto the surface using a $2 \mathrm{~mL}$ micrometre syringe (Gilmont). The roll-off angles were measured by tilting the stage 
until the droplet $(\sim 5 \mu \mathrm{L})$ started rolling off from the surface. Averages from at least six independent measurements were reported. The surface tension of probe liquids was evaluated using the pendant drop method, and all values fell within the range of previously reported literature values.

Microscopy. The surfaces were imaged by scanning electron microscopy (SEM) using a Philips XL30 scanning electron microscope at 5-10 kV. Energy-dispersive X-ray scattering was used to obtain the elemental mapping of various elements at the surface. Tapping-mode atomic force microscopy was conducted using a Veeco Innova instrument with Veeco TESPA tips.

Surface chemistry. The surface chemistry of the coatings was confirmed by X-ray photoelectron spectroscopy (Kratos Axis). The operation condition of monochromatic Al-K $\alpha \mathrm{X}-$ ray source was $10 \mathrm{kV}$ and $10 \mathrm{~mA}$. A take-off angle of $\sim 65^{\circ}$ was chosen to collect the photoelectrons.

Transparency. A Varian Cary 50 Bio UV-Vis Spectrometer was used to obtain transmittance data in the 300-900 $\mathrm{nm}$ range at a scanning rate of $10 \mathrm{~nm} \mathrm{~s}^{-1}$. Averages of three independent measurements were reported. Fig. 1d and e insets were obtained by placing the sprayed PDMS discs atop a periodic table and the virtual keyboard of a smartphone, respectively. The numbers on the smartphone display underneath the PDMS discs correspond to the coating time used. Data sets were plotted using the transmittance values at a wavelength of $600 \mathrm{~nm}$ unless otherwise stated.

Slope test. Liquid droplets were released on the coated fabric surface that was placed on a slope of $18^{\circ}$ or $37^{\circ}$. The rolling process was recorded, analysed, and compared with that predicted from the ideal friction-free model. 
Self-healing. Coated fabrics were first subject to $\mathrm{O}_{2}$ plasma treatment using a Harrick Plasmaflo plasma cleaner for $10 \mathrm{~s}$ to decompose the surface chemistry of the coating, consequently converting the surface into a complete wetting surface. Contact angles and roll-off angles were measured again after heat treatment at $120{ }^{\circ} \mathrm{C}$ for $10 \mathrm{~min}$ or storage at ambient temperature $(20$ $23{ }^{\circ} \mathrm{C}$ ) for $>24 \mathrm{~h}$. The coated Anticon fabrics can be $100 \%$ biaxially stretched for more than 1,000 times without destroying the woven structure or the binding of the coating to the substrate. For facially coated polyester fabrics, biaxial stretch will significantly increase the pore size and thus make any non-coated fibres accessible from the surface, leading to wetting of contacting liquids at corresponding elongation ratios. After releasing the fabric $(0 \%$ elongation $)$, the facial coating will again form a robust shield that prevents wetting.

Freezing retardation. The substrates were placed on a Peltier thermoelectric cold plate (TE Technology) that was precooled and kept at desired temperatures (e.g., $\left.-20^{\circ} \mathrm{C}\right)$. Then, a water droplet $(\sim 10 \mu \mathrm{L})$ was carefully placed on top of the surface. The freezing process was recorded using a Canon camera.

Ice adhesion test. An ice cube $\left(1 \times 1 \times 1 \mathrm{~cm}^{3}\right)$ was first prepared prior to the test. The substrate was precooled on a Peltier plate for $3 \mathrm{~min}$ at desired temperature $\left(-20^{\circ} \mathrm{C}\right.$ unless specified elsewhere). The ice cube was placed on the precooled substrate and kept for $3 \mathrm{~min}$. The movement of force gauge (Mark-10) was controlled by a syringe pump. The ice adhesion strength was given when the gauge pushes the ice to move. $T_{\text {ice }}$ is the maximum force that shears off the ice cube. More than 6 times of successive measurements were performed on each type of substrates. 
Durability testing. Droplet impact was carried out by dripping liquid droplets at a certain distance (10-300 mm) above the substrates. The roll-off angles of the hot (up to $100^{\circ} \mathrm{C}$ ) liquids (e.g., water and $n$-octane) and corrosive concentrated acids and bases were measured to examine the surface repellence under harsh conditions. The coated fabrics could endure the scratch test, $>$ 99 min sonication test, $>24 \mathrm{~h}$ washing test, $>100,000$ drop impacting test, $>1,000$ stretch-release cycles, $>100$ self-healing cycles and $>100$ ice removal tests with negligible changes both in liquid repellence and surface morphology (see Supplementary Information for details). In addition to the stretch test, the peel and shear tests were conducted to characterize the mechanical durability of the surface. In the peel test, a tape was placed across a fabric strip and then different weights $(20-100 \mathrm{~N})$ were applied statically for $60 \mathrm{~s}$ before peeling off the tape. In the shear test, the fabric that was placed directly underneath the loads was manually pulled forward at a speed of $\sim 1 \mathrm{~cm} \mathrm{~s}^{-1}$ until the load was left behind. The abrasion durability was conducted on a Martindale abrasion tester using a plain fabric as the abradant, and a pressure of $9 \mathrm{kPa}$ was applied. Contact angle and roll-off angle measurements, or SEM images were collected after these tests.

High-speed imaging. High-speed movies were obtained using a Fastec Hispec1 camera.

\section{Acknowledgments}

This research was conducted jointly and funded by the National Natural Science Foundation of China (NSFC, Grant nos. 51703056, 21707031, 21606081, 21527810, 21521063, 21575036, 21307029, 21221003, 21205034, and 21190041), National Key Basic Research Program (Project no. 2011CB911000), China Postdoctoral Science Foundation (Project no. 2016M602402), China Scholarship Council (File no. 201606130022), Natural Science Foundation of Hunan Province of 
China (Project no. 2018JJ3028), Horizon 2020/European Union (Grant agreement no. 745676), Australian Research Council (ARC) Centre of Excellence in Convergent Bio-Nano Science and Technology (Project no. CE140100036), and the ARC under the Australian Laureate Fellowship scheme (Grant no. FL120100030).

\section{Author contributions}

R.G. and S.P. conceived the ideas, and with the help of W.X., J.J., and F.C., designed and conducted the experiments, and analysed the data. All authors discussed and interpreted the results and contributed to the writing of the paper. R.G. and S.P. contributed equally to this work.

\section{Competing interests}

The authors declare no competing financial interests.

\section{Additional information}

Data supporting the findings of this study are available within the article (and its supplementary information) and from the corresponding authors upon reasonable request. 


\section{Figure captions}

Fig. 1 | Superrepellent, transparent PFTS/BCA coatings. a-b, Scanning electron microscopy (SEM) images of transparent superrepellent coatings. Scale bars are 50 and $5 \mu \mathrm{m}$, respectively. The insets show the contact of a millimetre-sized oil droplet with the coating (a) and the typical inverted hierarchical trapezoidal structure at the cross section (b). Scale bars in the insets of a and $\mathbf{b}$ are $5 \mathrm{~mm}$ and $5 \mu \mathrm{m}$, respectively. c, Molecular structure of chemicals used to form the coating. Alkyl groups and the fluorinated segments are shown in blue and red, respectively. d, Liquids of different surface tensions (THF, tetrahydrofuran; DMF, dimethylformamide; HF, hydrofluoric acid) residing on the transparent coatings. Scale bar is $5 \mathrm{~mm}$. e, Film transmittance as a function of coating time. The inset shows nine transparent discs spray-coated for different times (1-9 min) indicated by the numbers shown on the keyboard of a mobile phone display. Scale bar is $5 \mathrm{~mm}$. f, Images illustrating an ultralow surface tension oil droplet ( $n$-hexane, initial droplet radius $R_{0} \approx 1 \mathrm{~mm}$ ) bouncing on a horizontally placed spray-coated substrate. Time intervals are $\sim 6 \mathrm{~ms}$. Scale bar is $5 \mathrm{~mm}$. 
Fig. 2 | Near friction-free rolling of droplets on PTFS/BCA coatings. a, SEM image of a PTFS/BCA-coated fabric. Scale bar is $500 \mu \mathrm{m}$. b, Side and top views of droplets of pentane, THF, DMF, and water. The surface chemistry is also shown. Scale bar is $5 \mathrm{~mm}$. c, Typical hierarchical structure of a superficial fibre in (a). Scale bar is $10 \mu \mathrm{m}$. d, Superrepellency to various liquids (see Supplementary Table 2 for details). $\mathbf{e}-\mathbf{f}$, Top, middle, and bottom images in (e) respectively show a drop of $n$-pentane beading on the freshly coated surface, and penetrating the fabric after exposure to plasma treatment, but recovering the original superrepellency through annealing. Scale bar is $1 \mathrm{~mm}$. Multiple cycles of self-healing are shown in (f). The error bars are standard deviations obtained from at least 6 independent measurements. $\mathbf{g}-\mathbf{h}$, Rolling of an $n$ pentane droplet is illustrated in $(\mathbf{g})$. The rolling trajectory of the droplet (red solid circles) with ideal friction-free rolling (blue line) and predicted rolling, which is hindered by the contact angle hysteresis (CAH; red line), are shown in (h); the two plot sets correspond to tilt angles of $\alpha=18^{\circ}$ and $37^{\circ}$, respectively. i, Images illustrating an $n$-pentane $\operatorname{droplet}\left(R_{0} \approx 1 \mathrm{~mm}\right)$ bouncing on the coated fabric. Time interval is $\sim 6 \mathrm{~ms}$. Scale bar is $5 \mathrm{~mm}$. 
Fig. 3 | Stretch-responsiveness and extrusion of bouncing droplets. a-c, SEM images showing partially coated polyester fabrics that are uniaxially stretched along (a) or perpendicular (b) to the yarn, or biaxially stretched (c). Scale bar is $500 \mu \mathrm{m}$. d, Partially coated fabric displaying superomniphobicity at the released state and superomniphilicity subject to twofold biaxial stretching. Scale bar is $200 \mu \mathrm{m}$. e, Dependence of liquid contact angle and robustness factor $A^{*}$ on biaxial elongation of the fabric (see Supplementary Figs. 17 and 18 for more details). f, Restorability of $n$-pentane contact angles during release-stretch testing cycles. $\mathbf{g}$, Extruded jumping satellite droplet after an impinging $n$-pentane droplet bounces off the fully PTFS/BCA-coated fabric. Scale bar is $500 \mu \mathrm{m}$. 
Fig. 4 | Superrepellency even to sub-10 $\mathrm{mN} \mathrm{m}^{-1}$ liquids. a, Roll-off angles of liquids on the coated fabric surface (see Supplementary Fig. 25 and Supplementary Table 2 for more details). The ultra-low roll-off angles can be ascribed to superrepellence and/or other air cushioning effects. b, Beading, rolling and bouncing behaviour of liquid nitrogen upon impact on a coated print paper. The inset exemplifies the onset of liquid nitrogen impact. See Supplementary Figs. 26 and 27 for more details. Scale bar is $2 \mathrm{~cm}$. 